\title{
A Student Approach to a Mathematical Simulation of a Racing Electric Vehicle
}

\author{
Fernando Daniel León-Cázares and Daniel Xoconostle-Luna* \\ Instituto Tecnológico y de Estudios Superiores de Monterrey Campus Estado de México \\ *Students: fernando.leon.cazares@gmail.com,dxoconos@hotmail.com \\ Mentor: jorge.mendoza@itesm.mx
}

\begin{abstract}
A program was developed using the software Mathematica to simulate the dynamical behavior of an electric racing car, an electrathon. In conjunction with experimental data it is focused to allow the Borregos-CEM Racing Team to decide which settings have to be adjusted in order to increase the velocity of the racing car while decreasing its energy consumption, i.e. the current demanded to the batteries.
\end{abstract}

\section{KEYWORDS}

model, electric, racing, vehicle, dynamic, simulation, electrathon

\section{INTRODUCTION}

Electrathon began in England in 1978. Then it spread to Australia in 1980, the U.S.A. in 1990, and to Mexico in $1993 .^{1}$ After two years in Mexico it became an open championship to all universities and private participants. As of 2012, the championship involved 38 cars that ran with DC motors and commercial lead-acid batteries; the seven races were contested in several states across the Republic. While the future waits with great promises of electric and battery technologies, in the Borregos-CEM Racing Team, we are producing an EV (Electric Vehicle) beyond the championship's expectations. (From this point forwards, Team is an abbreviation of Borregos-CEM Racing Team)

To construct a car that rolls is quite easy, but to build a proper Electrathon there has to be an active awareness of the many details that are a natural part of the car. Then, to fashion the best possible racing EV there ought to be a CAD (Computer Aided Design), a CAE (Computer Aided Engineering) and, in the Team's case, the creation of a mathematical model of the car dynamics that is understandable to all.

Such a computer program was developed using the software Mathematica, to simulate the dynamical behavior of an Electrathon, which raced with DC (Direct Current) until 2012. Since the races last 90 minutes and allow a change of batteries, the championship focus is the optimization of the energy usage and limiting weight of the batteries. Hence, efficiency, defined as the ratio between the distance traveled and the electric charge consumed, remains as the most important factor to optimize. Thus, the whole idea of elaborating a theoretical model is to enhance the car's performance in the championship, which means an improvement in energy consumption. Because of this, the model is by now in its first steps to be a complete platform that includes all the dynamical properties of an EV, but that is flexible enough to be modified so that it successfully simulates a gasoline car.

The theoretical model in which the software is based was developed in order to obtain the required equations. This considers mechanical and electrical parameters that affect the vehicle's performance and may be optimized so that its velocity increases while its consumed charge decreases. Afterwards, it is explained how experimental data was obtained and compared with the simulations to validate the theoretical model previously mentioned. 


\section{THEORETICAL MODEL}

First of all a briefly explanation of the car to which the program was developed is given. Its powertrain consists of an electric DC motor, a roller chain, two sprockets, the rear shaft and both rear wheels. There is also a device ${ }^{2}$ that enables the slower rear wheel to lose traction in a curve so that the car can turn properly, as well as to completely avoid the power transmission when the angular velocity of the wheels is higher than the one delivered by the shaft.

The electric system has an electronic adjustable controller that allows the user to set a maximum output current as a percentage of the demanded by the motor in normal conditions. Once the motor curves of torque and current versus angular velocity are interpolated into the program, it is possible to obtain the force applied to the vehicle $F_{m}$ as

$$
F_{m}(v)=\frac{c l}{s r_{w}} * T_{m}\left(\frac{30 v}{\pi s r_{w}}\right),
$$

Equation 1.

where $c l$ is the controller limit (it can vary from 0 to 1 ), $s$ is the sprocket ratio of speed amplification, $r_{w}$ the wheel radius and $v$ the linear velocity of the vehicle. The function $T_{m}(\eta)$ is the interpolation of the motor's curve of torque versus angular velocity $\eta$ (in $\mathrm{N} \bullet \mathrm{m}$ and $\mathrm{rpm}$ respectively). Equation (1) is obtained by transmitting the motor's torque output to a linear force acting directly on the car, as well as by developing the necessary dimensional analysis to express all the parameters using the International System of Units.

Even though the mass of the vehicle remains unchanged during the competition it is a really important parameter. Also, its geometry should be taken into account due to the effect of the drag force $F_{d},{ }^{3}$ which can be approximated for a vehicle as

$$
F_{d}(v)=\frac{1}{2} C_{d} \rho A v^{2},
$$

Equation 2.

where $C_{d}$ is the drag coefficient, $\rho$ the air's density (considered as a constant equal to $\left.1.2041 \mathrm{~kg} / \mathrm{m}^{3}\right), v$ the linear velocity of the car, and $A$ its frontal area. Considering a parameter $\beta=C_{d}$ $A$, equation (2) becomes

$$
F_{d}(v)=0.60205 \beta v^{2} .
$$

Equation 3.

Friction due to mechanical interactions between the components of the vehicle represents also a force that acts against motion. However, it is neglected as it is really small when compared with the drag force. ${ }^{4}$

The last force taken into account is the one produced by gravity, $F_{g}$, when the slope is other than zero. It can be expressed as

$$
F_{g}=m g \sin (-\alpha),
$$

Equation 4.

where $m$ is the vehicle's mass and $\alpha$ the angle between a horizontal plane and the one with the slope (an angle $\alpha>0$ means that the car is going up).

Given the 2nd Newton's Law, the car's acceleration $a(v)$ can be calculated as

$$
a(v)=\frac{F_{m}(v)-F_{d}(v)+F_{g}}{m},
$$

Equation 5.

and substituting equations (1), (3) and (4) into (5), as well as considering that $a(v)=d v / d t$, we get the ordinary differential equation

$$
v^{\prime}(t)=\frac{c l}{s m r_{w}} * T_{m}\left(\frac{30 v(t)}{\pi s r_{w}}\right)-\frac{0.60205 \beta v(t)^{2}}{m}+g \sin (-\alpha), \quad \text { Equation } 6 .
$$


with the initial velocity $v_{0}$ known

$$
v(0)=v_{0},
$$

Equation 7.

in terms of $v(t)$, where $t$ is the time. This can be solved numerically in Mathematica no matter what form has the motor's curve. Given the function $v(t)$ it is possible to find the displacement $x$ traversed by the vehicle as a function of time as

$$
x(t)=\int_{0}^{t} v(t) d t
$$

Equation 8.

The total time $t_{f}$ necessary for the car to complete a straight segment of a circuit can be calculated by solving for the time the equation

$$
x(t)=\frac{x_{f}}{\cos (\alpha)},
$$

Equation 9.

where $x_{f}$ is the horizontal length of that segment. It is important to note that for a circular path it is necessary to change this length $x_{f}$ for $r^{*} \theta$, where $r$ is its radius and $\theta$ its angle of displacement. Just for this case it is also possible to obtain a function of the centripetal acceleration $a_{c}$ in terms of time as

$$
a_{c}(t)=\frac{v(t)^{2}}{r} .
$$

\section{Equation 10.}

After this analysis it is possible to determine the dynamical behavior of an accelerating electric vehicle. Nevertheless, it is also an objective of this program to obtain a function of the charge demanded to the batteries. In order to accomplish this, it is necessary to interpolate a function of the current $I_{m}\left(T_{m}(\eta)\right)$ in terms of the motor's torque. This information is given by its manufacturer. Taking the controller into account, as well as the relationship between the vehicle's angular and linear velocity, a function in terms of the last one, $I_{l v}$, can be stated as

$$
I_{l v}(v)=c l * I_{m}\left(T_{m}\left(\frac{30 v}{\pi s r_{w}}\right)\right)
$$

Equation 11.

To get the instantaneous current at any time it is just required to evaluate in a function $I(t)$ in terms of the time as

$$
I(t)=I_{l v}(v(t)),
$$

Equation 12.

and finally the total charge $Q$ consumed in a segment of a circuit is obtained as

$$
Q=\int_{0}^{t_{f}} I(t) d t
$$

Equation 13.

Given all these equations, a complete circuit can be run, taking into account that the initial velocity of each segment is the same as the one obtained at the last instant of the previous one. After adding each time and charge consumed, many laps can be rapidly simulated. It is also possible to develop the model with no torque from the motor, so that there are segments in which the pilot does not accelerate. 


\section{EXPERIMENTAL}

The motor used was a Briggs and Stratton Etek ${ }^{5}$ at $36 \mathrm{~V}$. However, as it may be seen, the manufacturer's data sheet only shows the information for the range from nearly 2125 to $2592 \mathrm{rpm}$. Also, a maximum torque of $47.73 \mathrm{~N} \cdot \mathrm{m}$ is reported, as well as a linear relationship between this property and the current demanded of $0.1288 \mathrm{~N} \cdot \mathrm{m} / \mathrm{A}$. The controller used was an Alltrax Axe $48455^{6}$ For the simulations, a linear interpolation for the unknown range of angular velocities was used. The vehicle, including the batteries and the pilot, had a mass of $192 \mathrm{~kg}$, its wheels had a 5 inch radius and it was possible to estimate the coefficient $\beta$ with the help of the software Ansys Fluent running a simulation with the vehicle's CAD. This simulation's output showed that the car had a drag coefficient of 0.24018 and an estimation of a $1.444 \mathrm{~m}^{2}$ frontal area; of which $0.622 \mathrm{~m}^{2}$ correspond to the bodywork. However, the remaining exposed area has a higher drag coefficient. Hence the calculated $\beta=0.346$ for the bodywork had to be increased to include the rougher surface of the rest of the car. Several simulations were run to fit this parameter with the results of a previous race. As it can be seen in Figure 1, a coefficient $\beta=0.8 \mathrm{~m}^{2}$ proved to be the best fit, so that further simulations were closer and more accurate.

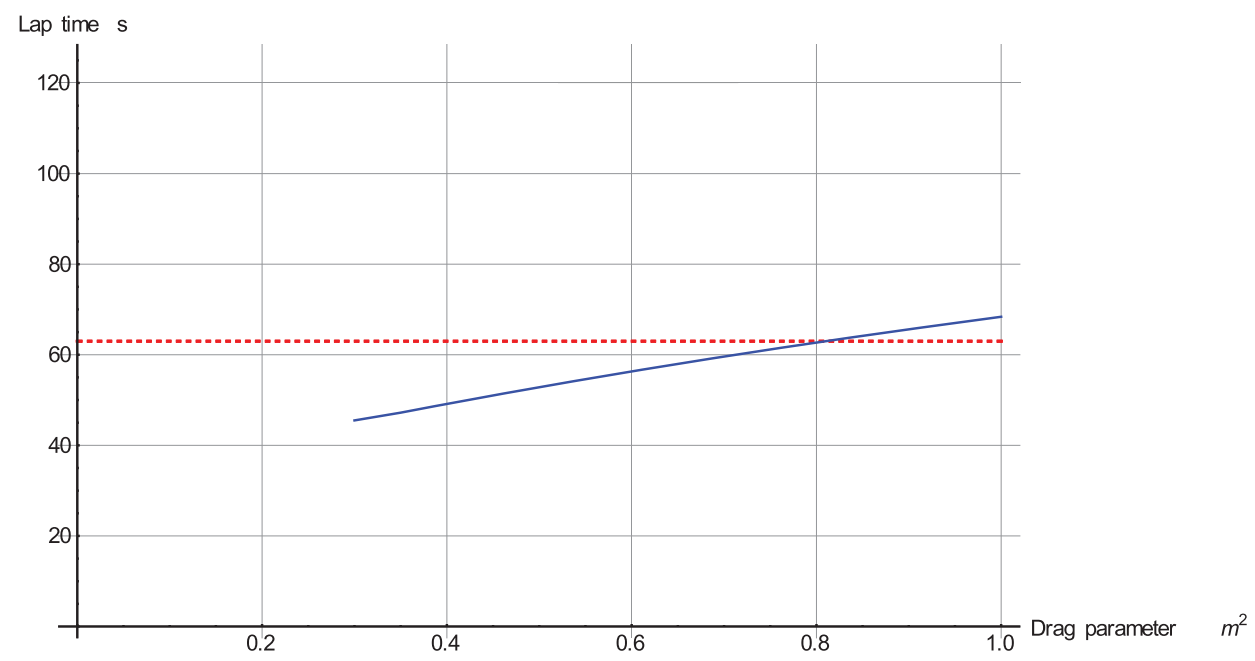

Figure 1. Experimental fit of the drag parameter $\beta$ from a previous race in Kartódromo La Mina, Toluca, Mexico at August 26th, 2012 for the average lap time (dashed line) of $63.03 \mathrm{~s}$. The solid line represents the simulations run by the software for different values of $\beta$.

To further validate the program it was necessary to gather as many reliable experimental data as possible. Measures were taken from two sources: straight line sprints and during the races. For the sprints, the car was equipped as it would be for any race and a set distance is measured from 0 to 200 meters with marks every 50 meters. The test began with a pre-set sprocket ratio of 0.5357 and the controller limit was changed after each sprint to $0.2,0.3,0.5$ and 0.7. Afterwards, the same values were tested for a ratio of 0.4643 . This test was designed to run the car in a controlled environment (climate conditions may vary even during a test) and to know how these parameters affect its velocity.

In the championship, it was possible to base the decision of the parameters' values to use just in the last race. Each circuit was modeled by segmenting it in many parts, all of them straight lines or perfect arcs of a circle, and measuring them with the help of Google Earth. This software was also helpful for determining the angles of each division's different slopes as it offers a complete height profile of the terrain. However, even though it was difficult to take a continuous measurement of the velocities and currents, we managed to take the times of each lap to compare them with the 
expected ones. There are many factors, such as the pilot and the other contestants, which make it complicated to quantitatively obtain a constant performance. Even when the manufacturers give the theoretical total charges of the batteries, ${ }^{7}$ it varies depending on the discharge speed ${ }^{8}$ and their voltage drop makes it difficult to determine when they can be considered as depleted. Also, as the voltage decreases, the overall performance of the car gets continuously modified. This is why we could only appreciate a qualitative change with respect to the previous races.

\section{RESULTS}

To validate the accuracy of the software model, we used the gathered data of the tests (Table 1) to compare it against the results that the program was predicting (Table 2).

\begin{tabular}{|l|l|l|l|l|l|l|l|l|}
\hline \multicolumn{8}{|c|}{$\begin{array}{l}\text { Experimental data } \\
\text { Length } \\
{[\mathrm{m}]}\end{array}$} & \multicolumn{9}{|c|}{\begin{tabular}{l}
$\mathrm{s}]$ \\
\cline { 2 - 9 }
\end{tabular}} & $\begin{array}{l}\mathrm{s}=0.5357 \\
\mathrm{cl}=0.2\end{array}$ & $\begin{array}{l}\mathrm{s}=0.5357 \\
\mathrm{cl}=0.3\end{array}$ & $\begin{array}{l}\mathrm{s}=0.5357 \\
\mathrm{cl}=0.5\end{array}$ & $\begin{array}{l}\mathrm{s}=0.5357 \\
\mathrm{cl}=0.7\end{array}$ & $\begin{array}{l}\mathrm{s}=0.4643 \\
\mathrm{cl}=0.2\end{array}$ & $\begin{array}{l}\mathrm{s}=0.4643 \\
\mathrm{cl}=0.3\end{array}$ & $\begin{array}{l}\mathrm{s}=0.4643 \\
\mathrm{cl}=0.5\end{array}$ & $\begin{array}{l}\mathrm{s}=0.4643 \\
\mathrm{cl}=0.7\end{array}$ \\
\hline 50 & 12.4 & 10.3 & 7.6 & 6.7 & 11.8 & 9.2 & 6.9 & 6.7 \\
\hline 100 & 19 & 15.3 & 11 & 10 & 17.5 & 13.6 & 10.5 & 10 \\
\hline 150 & 23.9 & 19.1 & 14.2 & 13.1 & 21.8 & 16.8 & 13.8 & 13.5 \\
\hline 200 & 27.1 & 21.6 & 16.6 & 15.9 & 25.6 & 20.3 & 17.1 & 16.8 \\
\hline
\end{tabular}

Table 1. Experimental data of the sprints for length intervals of $50 \mathrm{~m}$. $s$ is the sprocket ratio of speed amplification and $c l$ the controller limit.

\begin{tabular}{|c|c|c|c|c|c|c|c|c|}
\hline \multicolumn{9}{|c|}{ Simulation data } \\
\hline \multirow{2}{*}{$\begin{array}{l}\text { Length } \\
{[\mathrm{m}]}\end{array}$} & \multicolumn{8}{|c|}{ Time $[\mathrm{s}]$} \\
\hline & $\begin{array}{l}\mathrm{s}=0.5357 \\
\mathrm{cl}=0.2\end{array}$ & $\begin{array}{l}\mathrm{s}=0.5357 \\
\mathrm{cl}=0.3\end{array}$ & $\begin{array}{l}\mathrm{s}=0.5357 \\
\mathrm{cl}=0.5\end{array}$ & $\begin{array}{l}\mathrm{s}=0.5357 \\
\mathrm{cl}=0.7\end{array}$ & $\begin{array}{l}\mathrm{s}=0.4643 \\
\mathrm{cl}=0.2\end{array}$ & $\begin{array}{l}\mathrm{s}=0.4643 \\
\mathrm{cl}=0.3\end{array}$ & $\begin{array}{l}\mathrm{s}=0.4643 \\
\mathrm{cl}=0.5\end{array}$ & $\begin{array}{l}\mathrm{s}=0.4643 \\
\mathrm{cl}=0.7\end{array}$ \\
\hline 50 & 12.628 & 10.444 & 8.259 & 7.101 & 11.918 & 9.89 & 7.864 & 6.792 \\
\hline 100 & 18.673 & 15.533 & 12.4 & 10.745 & 17.732 & 14.823 & 11.929 & 10.45 \\
\hline 150 & 23.779 & 19.87 & 15.98 & 13.952 & 22.69 & 19.077 & 15.548 & 13.88 \\
\hline 200 & 28.459 & 23.872 & 19.318 & 17.042 & 27.267 & 23.036 & 19.066 & 17.276 \\
\hline
\end{tabular}

Table 2. Simulated data of the sprints for length intervals of $50 \mathrm{~m} . s$ is the sprocket ratio of speed amplification and $c l$ the controller limit.

The average error obtained from comparing tables 1 and 2 was about $6.85 \%$. This is relatively low, and it may occur due to the lack of the motor's information at low speeds, as well as to measurement errors, battery discharging from one test to another, wind effects and different tire temperatures, among others.

The race took place in a parking lot at ITESM Guadalajara and the average lap time was of 44 seconds. The sprocket ratio used was of 41.67 , while the controller limit was set to $25 \%$. On the other hand, the simulation output for 4 laps was the following one:

Lap 1: Time $=51.6364 \mathrm{~s}$; charge $=2006.55 \mathrm{C}$

Lap 2: Time $=43.5078 \mathrm{~s}$; charge $=1234.42 \mathrm{C}$

Lap 3: Time $=43.5044 \mathrm{~s}$; charge $=1233.78 \mathrm{C}$

Lap 4: Time $=43.5044 \mathrm{~s}$; charge $=1233.78 \mathrm{C}$ 
As it may be seen, the time per lap reaches a minimum after several iterations, that fact that does not apply for real life. The simulation error obtained was of about $1.126 \%$ in comparison with the measured times. Figure 2 shows a graph of velocity versus time, where it is possible to appreciate how terrain elevations and segments in which the car is not allowed to accelerate make the profile obtained a variable one.

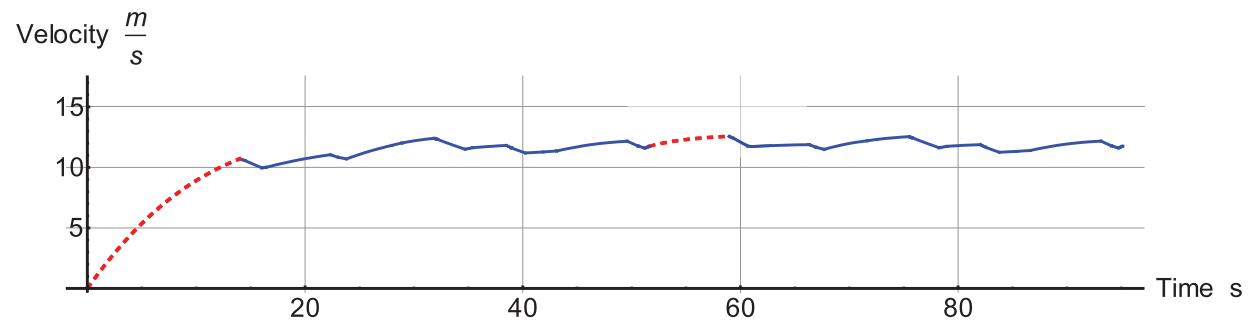

Figure 2. Simulation output for the first two laps of the championship's race in Instituto Tecnológico y de Estudios Superiores de Monterrey Campus Guadalajara at November 3rd, 2012. Dashed lines represent the first segment of each lap.

Regarding the electric performance, we were unable to accurately measure the current at each point of the circuit. However, we could ensure that the batteries never reached a critical point and the car maintained a regular time in each lap.

This means that the developed program is useful to predict the results with ever increasing accuracy, as long as the feedback is fresh data. A major point of the program is that by running a large number of simulations, with different controller limits and sprocket ratios, a decision matrix can be made so that the Team can decide which values is the optimum to use in each race. Even though physical testing was sought every day, due to the time required to charge the batteries and the risk of injuring the vehicle, the simulations provided the best source of information we could acquire while not in a race.

\section{CONCLUSIONS}

This present model, as shown in the results, has proved to be a useful tool to optimize some parameters of the vehicle and to show how each of them affect its dynamical and electrical behavior. However, there is some variation between the real performance and the one predicted by the simulations due to many factors. Even though the lack of information regarding the motor curves and possible variations from its theoretical operation represent a serious inconvenience, it is possible to circumvent this issue by adequately testing the used motor. Regarding the $\beta$ coefficient, it was possible to fit this value to a real approximation by using data from previous races. With the necessary experimentation and simulations it is possible to find a more accurate value of this parameter. Variable battery voltages during the races due to discharge make it impossible to have a constant behavior, resulting in changes from the simulation outputs. Nevertheless, it is possible to neglect these variations as the voltage drop becomes important only after long periods of time.

Even while this program is still being improved, it is already offering good results the Team can exploit. As it is shown in the results, this software can be really useful to predict the behavior of the car and to determine the parameters to be used in every race, taking into account the terrain elevation profile. By this it is possible to adjust the simulation to the given conditions in each race. The obtained results in the sprint tests, in conjunction with the ones in the circuits, proved to have a percentage error of less than $7 \%$.

Among the contemplated modifications lies an algorithm to automatically determine where and when the pilot should stop accelerating in order to take a curve at the maximum possible velocity. Also, this model could predict the behavior of many types of vehicles as it may be upgraded to 
work with AC motors and even fuel engines. With these modifications accurate prediction could be possible just by establishing the corresponding curves for torque and current (or fuel consumption rate) vs. angular velocity. For the latter case a variable mass should be also taken into account. These are some of the reasons why this program could become a very useful tool for analyzing many types of vehicles, focusing in racing applications.

\section{ACKNOWLEDGEMENTS}

We truly thank professors Jorge Aguirre and José Luis Gómez for encouraging us to develop the model and to publish it. We also thank each member of the Team for their constant collaboration.

\section{REFERENCES}

[1] Toyota-Electratón-LTH. (2013) History, retrieved 6 June 2013, from http://www.electraton.com. $\mathrm{mx} /$ historia.htm.

[2] SKF. (2013) Needle roller and cage assemblies, retrieved 6 June, 2013, from http://www.skf. $\mathrm{com} / \mathrm{ca} /$ en/products/bearings-units-housings/roller-bearings/needle-roller-bearings/needle-roller-and-cage-assemblies/index.html.

[3] White, F. (1998) Fluid Mechanics, 4th ed., McGraw Hill, New York, NY.

[4] Bettes, W. (1982) The Aerodynamic Drag of Road Vehicles-Past, Present, and Future, Engineering and Science 45, 4-10.

[5] Stratton, B. a. (2001) Etek Electric Motor System, retrieved 6 June, 2013, from http://www.robotmarketplace.com/products/images/etekmanual.pdf.

[6] Alltrax. (2008) AXE Performance products used for series wound or permanent magnet electric motors, retrieved 6 June, 2013, from http://www.alltraxinc.com/Products_AXE.html.

[7] Balsas, A. y. F. d. (2012) Full Power FP-75-650, retrieved 6 June, 2013, from http://www.afbalsas. com/index.php/component/content/article/19-productos/acumuladores/full-power/291-full-powerfp-75-650.

[8] Pascoe, P. a. A., A.. (2004) VRLA Battery Discharge Reserve Time Estimation, Power Electronics, IEEE Transactions 19, 1515-1522.

\section{ABOUT THE STUDENT AUTHORS}

Fernando León-Cázares is a senior student in the area of mechanical engineering in Instituto Tecnológico y de Estudios Superiores de Monterrey Campus Estado de México. He was the Electrathon Borregos CEM Racing's captain for two years and he has participated in a research group in the same institute dealing with the mathematical modeling of a plasma nitriding process. Daniel XoconostleLuna is a mechatronics engineering student who began to work full time in 2013.

\section{PRESS SUMMARY}

An Electrathon is a custom built electric vehicle, similar in appearance to a Go-Kart but powered by an electric motor. We developed a mathematical model to simulate the performance of such vehicle under different conditions so that it is possible to predict its velocity, acceleration and energy consumption at any point of any specific track. This allows for the optimization of different parameters of the vehicle to build the best Electrathon possible. 